\title{
AUTOMATIC ROAD DETECTION OF SATELLITE IMAGES USING IMPROVED EDGE DETECTION
}

\author{
Supreet Kaur ${ }^{1}$, Seema Baghla ${ }^{2}$
}

Dept. of Computer Science, Yadavindra College of Engineering, Talwandi Sabo, Bathinda (Pb.) India ${ }^{1}$

$$
\text { supreet_kaur0222@yahoo.com }{ }^{1}
$$

Dept. of Computer Science, Yadavindra College of Engineering, Talwandi Sabo, Bathinda (Pb.) India ${ }^{2}$

$$
\text { garg_seema238@yahoo.co.in² }
$$

\section{ABSTRACT}

Road networks play an important role in a number of geospatial applications, such as cartographic, infrastructure planning and traffic routing software. Automatic and semi-automatic road network extraction techniques have significantly increased the extraction rate of road networks. The road detection performance is a critically affected by the noise or low frequency images. Road edge detection plays a significant role to detect the direction of the road and the specific location of obstacles, size and speed of obstacles in the road. In this paper, several road detection techniques are theoretically analyzed, and a new road detection technique is proposed using median filter. By comparing it with some well known techniques it is found that there exist many gaps in the techniques proposed so far. By comparing the proposed algorithm with proposed it is proved that the proposed algorithm does not provide artifacts were quite more in old techniques.

\section{Keywords}

Road images; Edge detection, Road detection, Satellite remote sensing

\section{Academic Discipline And Sub-Disciplines}

Computer Science and Engineering

\section{SUBJECT CLASSIFICATION}

Image Processing, Edge Detection.

\section{TYPE (METHOD/APPROACH)}

Experimental setup; Literary Survey

\section{Council for Innovative Research}

\author{
Peer Review Research Publishing System
}

\section{Journal: INTERNATIONAL JOURNAL OF COMPUTERS \& TECHNOLOGY}

Vol 10, No 4 


\section{INTRODUCTION}

Today satellite remote sensing systems [1]-[7] provide large volumes of data that are invaluable in monitoring Earth resources and the effects of human activities. Road feature extraction from remotely sensed images has been a long term topic of research and because of its complexity is still a challenging topic[6]. The ability of the next generation sensors to provide fine spatial resolution data has motivated the urgency for automated road extraction research.

Accurate and up-to-date road network information is essential for urban planning, automated road navigation, and emergency response applications [3]. Automated methods have the potential to improve the speed and utility for road mapping and are therefore highly desirable. Roads are only extracted in the regions around database roads. Road extraction is difficult in the presence of context objects such as buildings or trees close to the road, disrupting the appearance of the road or occluding it.

Many approaches for road extraction have been developed. However, only few approaches work in urban scenes which complicates the task of automatic road extraction [1]. The comparison of the two methods i.e. normalized cuts method and mean shift method is done in this literature. A normalized cut is a graph based method taking both local and global characteristic of the image [3]. The combination of the local and global aspects ignores noise, small surface changes and weak edges and producing extraction with most segments covering only a road area [8]. In this approach, only the boundaries are considered. The advantage of this method is that hard constraints are not needed to gain information about roads. This makes this method more conductive for automatic road extraction.

Mean shift method is a clustering technique used to classify data into different categories and does not require information about specific object and extracts road information exactly by object oriented method. In this method, data is segmented and these segments are analyzed to detect road - like and non- road segments.

\section{LITERATURE SURVEY}

A literature review goes beyond the search for information and includes the identification and articulation of relationships between the literature and our field of research. While the form of the literature review may vary with different types of studies, the basic purposes remain constant:

M. Rajeswari et al. [1] has evaluated the speed of growth of an urban area road is one of the fast information updating element during urban development. Road information extraction based on high resolution satellite images play an important role because roads affect city land usage. In this paper, two approaches for road network extraction for an urban are proposed.

J. Senthilnath et al. [2] has evaluated the the study of Urban population is growing so fast in India that planning officials are racing to keep up with urban development .Use of geographic information like satellite imagery helps urban planners manage the ever-changing urban environment accurately and efficiently. Roads are one of the most important features to be extracted from Satellite imagery for urban planning. Manual extraction of roads is operator dependent and timeconsuming task.

M. Butenuth et al. [3] introduce a paper in which they study road extraction of high resolution aerial images of suburban scenes based on segmentation using the Normalized Cuts algorithm. The aim of our project is the extraction of roads for the assessment of a road database, however, this paper is restricted to road extraction. The segmentation as our basic step is designed to yield a good division between road areas and the surroundings.

M.Rajeswari et al. [4] introduce a paper in which they study Automatic road network extraction based on high resolution satellite image for urban planning holds great potential for significant reduction of database development/updating cost and turnaround time. Satellite remote sensing has been recognized worldwide as an effective technology for the monitoring and mapping the urban development.

V.Parthasarathi et al.[5] has evaluated the study analysis of high resolution satellite images has been an important research topic for urban analysis. One of the important features of urban areas in urban analysis is the automatic road network extraction.

T Rajani Mangala and S G Bhirud [6] has evaluated the study of road information extraction from high resolution satellite images plays an important role because roads affect urban and rural land cover and usage. It is difficult and computationally intensive and expensive to extract roads due to presence of their road like features with straight edges.

O.Sharma et al. [7] introduce a paper in which they study that areal features are of great importance in applications like shore line mapping, boundary delineation and change detection. This research work is an attempt to automate the process of extracting feature boundaries from satellite imagery.

E. Karaman et al. [8] has evaluated the study to develop automatic road extraction algorithm in satellite images. As roads have different width and surface material characteristics in urban and rural areas, a modular approach for road extraction algorithm is desired. In this study, edge detection, segmentation, clustering and vegetation and land cover analyses are used. 
F.Chun ling and R. Yuan yuan [9] introduce a paper in which road detection methods are becoming very important part of vision applications and play a significant role in intelligent transportation system, which is the key technique of intelligent vehicle guidance. The road detection performance is an critically affected by the noise or low frequency images.

\section{GAPS IN LITERATURE}

By conducting the literature survey it has been found that most of persisting researches has neglected one of the following.
a) Noise
b) Restoration
c) Debluring

As we know that above parameters may decrease the performance of the road detection system. So, these parameters become the critical issue of road detection system.

\section{HOW ROAD DETECTION WORKS}

The past few years was marked by the development of researches that contribute to reach automatic road extraction which is perceived as a possible solution to prevent human errors in the quality evaluation process. There are various methods in the quality control which can replace the human operator. One of these methods includes Computer vision system. After hours of working the operator may lose concentration which in turn will affect the evaluation process. So a computer vision system proved to be more efficient at the level of precision and rapidity.

Here is the steps of proposed work have to be done for this research of road extraction.

\section{Step 1: Pre-Processing}

a) Classification: - dividing the image into two classes - roads and non-roads.

b) Grouping: - A nearest neighborhood grouping (NNG) operation is applied to the classified representation for smoothing the spectral response within the pixel's local neighborhood.

\section{Step 2: Filtering}

The method of road extraction in certain locations poses challenge because the spectral reflectance of some of the old buildings resembles the road surface. Such buildings from the clutter and these non-road structures need to be removed. A morphological opening operation is applied which identifies road blocks.

\section{Step 3: Segmentation}

Segmentation is the process of partitioning the digital image into multiple segments.

\section{Step 4: Road Extraction}

There are two methods for the extraction of road using satellite images

a) Normalized Cut Method

b) Mean Shift Method

\section{Step 5: Image Overlaying}

In order to illustrate the accuracy, the extracted road region using is converted into binary image format. This binary image is overlaid on the original panchromatic image leads to display the road topology by avoiding the complex noise element. In the overlaid image the thin lines indicate the road topology.

\section{Step 6: Performance analysis}

The automatically extracted roads are compared with manually traced reference roads to perform accuracy assessment. Since roads have linear features, it is possible to use all the data rather than just sample points to conduct the accuracy assessment.

\section{PROPOSED ALGORITHM}

Figure 1 is showing the flowchart of the proposed algorithm which contains information in steps:

Step 1: Input Road Image: Input any road image if it is in RGB then convert it into gray image

Step 2: Filtering: The gray image is filtered using median filter to remove noises that effects satellite images.

Step 3: Binarization algorithm: Convert the filtered image into binary image for easy and fast processing using binarization algorithm 


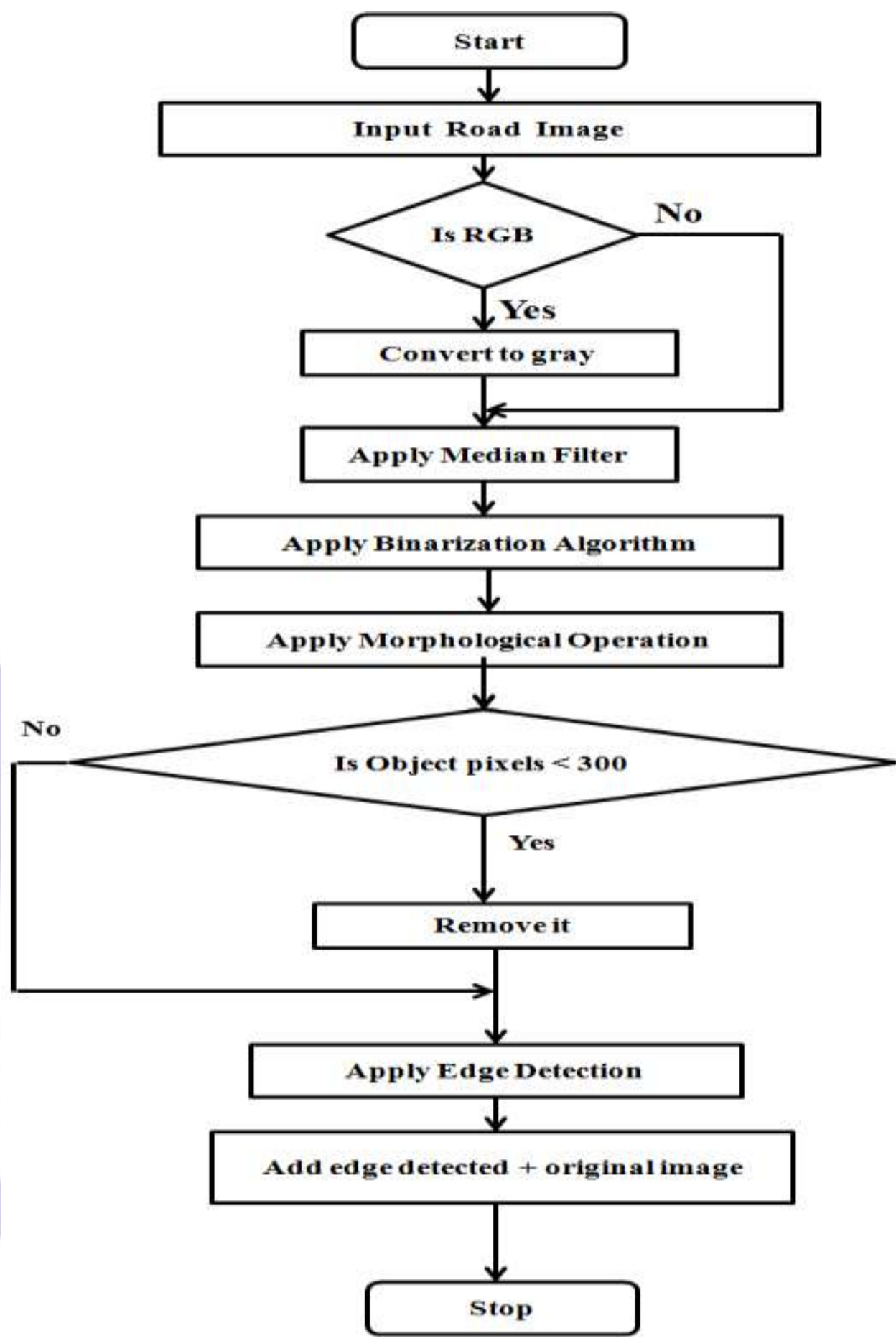

Fig. 1 Flowchart of proposed technique

Step 4: Morphological Operation: The binary image is still contains some unwanted pixels. One of the easiest ways to remove unwanted pixels from an image is by using morphological operations.

Step 5: Edge Detection: After applying the morphological operation we get the clean road image but it is important to obtain the edges on the roads on the image. Any edge detection algorithm can be applied i.e. canny edge detection, sobel edge detection, prewett edge detection etc. for edge detection.

Step 6: Overlaying the result: The final step is overlay the extracted road into the scalar image of the original image. Overlaying of the result helps to illustrate the accuracy of the road extraction. In the final image, the thin lines indicate the paths of roads in the image. 


\section{EXPERIMENTAL SETUP}

Table1. Input Test bed

\begin{tabular}{|l|l|l|l|}
\hline S.No. & Name & Format & Size (in KB) \\
\hline 1 & image1 & Jpg & 72.1 \\
\hline 2 & image2 & Jpg & 66.3 \\
\hline 3 & image3 & Jpg & 79.3 \\
\hline 4 & image4 & Jpg & 86.5 \\
\hline 5 & image5 & Jpg & 75.7 \\
\hline 6 & image6 & Jpg & 83.9 \\
\hline 7 & image7 & Jpg & 89.8 \\
\hline 8 & image8 & Jpg & 69.4 \\
\hline 9 & image9 & Jpg & 70.8 \\
\hline 10 & image10 & Jpg & 78.1 \\
\hline
\end{tabular}

\section{RESULTS}

By running the proposed and an existing algorithm by taking image1 at a time following results are taken. Old technique is proposed by F.Chun ling and R. Yuan yuan [9]. They have used different edge detection operators to detect roads.

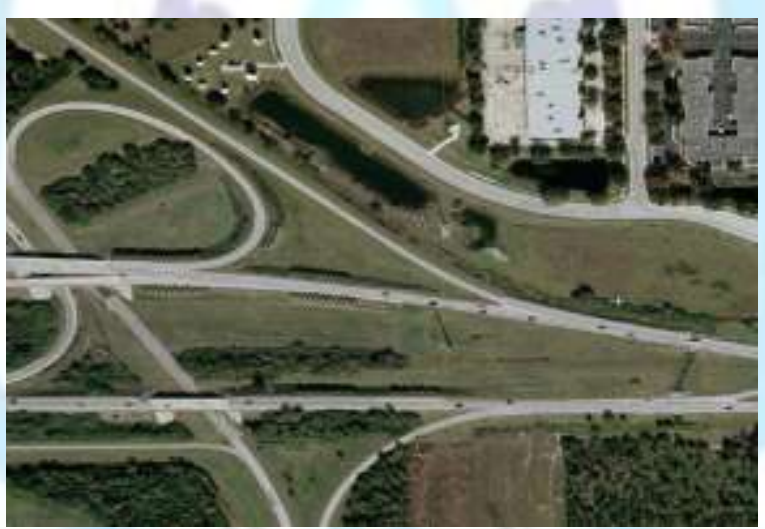

Fig. 2lnput image:

Fig2 shows an input image for both old and new technique. It is a satellite image showing some roads in white colour.

\section{Old Results}

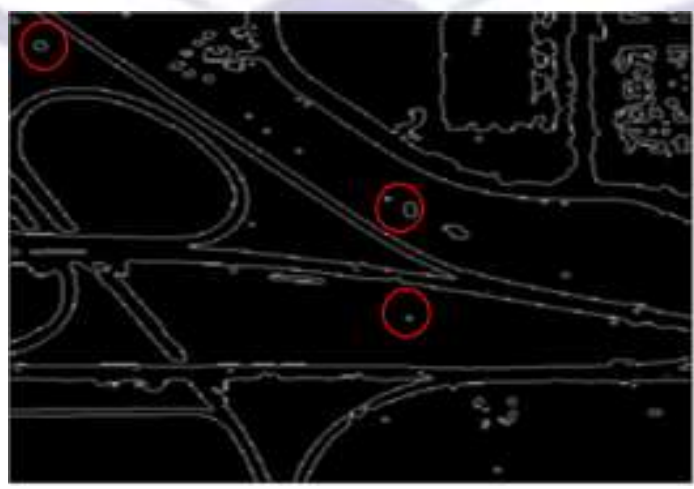

Fig. 3 Segmented Image using old technique

Fig. 3 shows the segmented image using old technique. There are so many unwanted objects which are indicated by red circle. So, the old technique is not provide efficient results. 


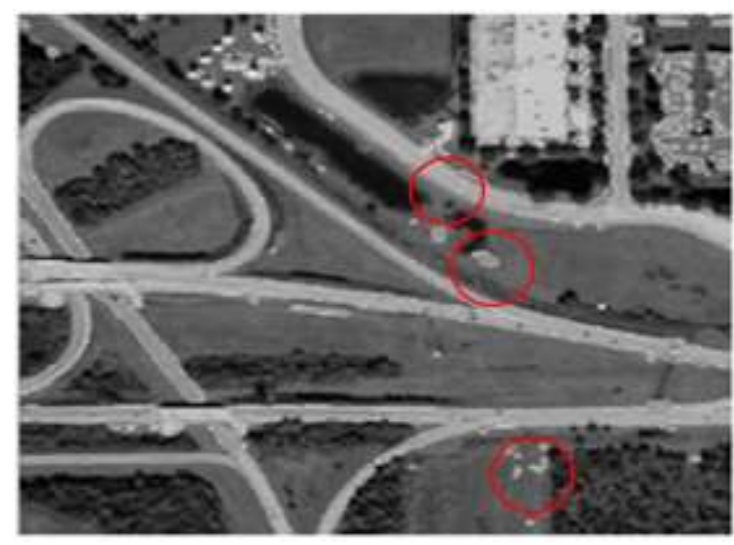

Fig. 4 Output Image using old technique

Fig.4 shows the output image using old technique in which red circles shows unwanted objects. It shows that there are so many artefacts which reducing the performance of existing technique.

\section{New Results}

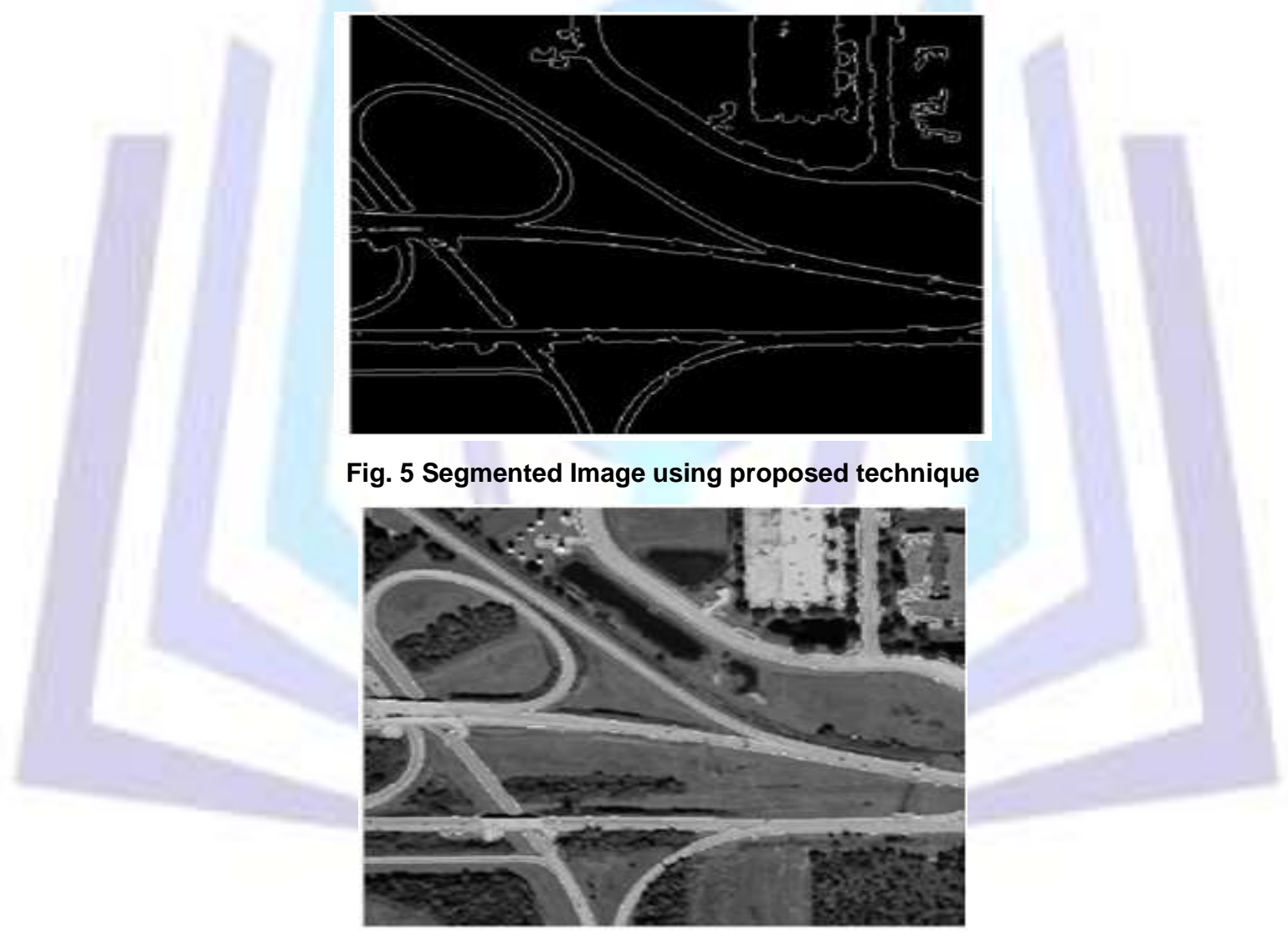

Fig. 6 Output Image using proposed technique

Fig. 5 shows the segmented image using proposed technique. It shows that there are no artefacts in proposed technique which indicates the better performance as compared to existing technique.

Fig. 6 shows the final output result image using proposed technique with better performance as compared to existing technique. There is no artefacts present in proposed technique.

\section{CONCLUSION}

Recently, road detection methods are becoming very important part of vision applications and play a significant role in Intelligent transportation system, which is the key technique of intelligent vehicle guidance. The road detection performance is a critically affected by the noise or low frequency images. To overcome this problem we have proposed a new algorithm which used median filter and removal of low intensity pixels to improve the results. By comparing the technique with old methods it is found that the proposed algorithm is proving better results than the old techniques. 


\section{REFERENCES}

[1] M.Rajeswari, K.S.Gurumurthy, S.N.Omkar, J.Senthilnath, L.Pratap Reddy, "Automatic extraction of road networks based on normalized cuts and mean shift method for high resolution satellite imagery", International journal of advanced engineering sciences and technologies, Vol.3, pp. 115-121.

[2] M.Rajeswari, K.S.Gurumurthy, S.N.Omkar, J.Senthilnath, L.Pratap Reddy (2011), "Automatic road extraction based on level set, normalized cuts and mean shift methods", International journal of advanced engineering sciences and technologies, Vol.8, pp. 250-257.

[3] A.Grote, M.Butenuth, C.Heipke (2007), "Road extraction in suburban area based on normalized cuts", International archives of photogrammetry, remote sensing and spatial information sciences, pp. 51-56.

[4] M.Rajeswari, K.S.Gurumurthy, S.N.Omkar, L.Pratap Reddy,(2011), "Automatic Extraction of Road Networks based on Normalized cuts and Mean shift method for high resolution satellite imagery", International journal of computer applications, Vol.18-No.7, pp. 10-16.

[5] V.Parthasarathi, DY.Pushpamitra, (2012), "Real-time implementation of automatic road extraction for high resolution satellite images using FPGA", Global journal for information technology and computer science, Vol.1 Issue. 1, pp. 1-6.

[6] T.R.Mangala, S.G.Bhirud, (2011), "Road network extraction from high resolution satellite images based on LSE, LBF model”, International journal of scientific and engineering research, Vol.2, Issue 10 pp. 1-6.

[7] O.Sharma, D.Mioc, F.Anton, (2008),"Polygon feature extraction from satellite imagery based on colour image segmentation and medial axis", The international archives of the photogrammetry, remote sensing ang spatial information sciences, Vol.38, pp. 235-240.

[8] E.Karaman, U.Cinar, E.Gedik, Y.Yardemci, U.Halici, (2012), "A new algorithm for automatic road network extraction in multispectral satellite images", Proceedings of the 4th GEOBIA, pp. 455-4598.

[9] Chun-ling Fan and Yuan-yuan Ren, (2010)," Study on the Edge Detection Algorithms of Road Image", IEEE, pp. 217 220.

[10] Spector, A. Z. 1989. Achieving application requirements. In Distributed Systems, S. Mullender

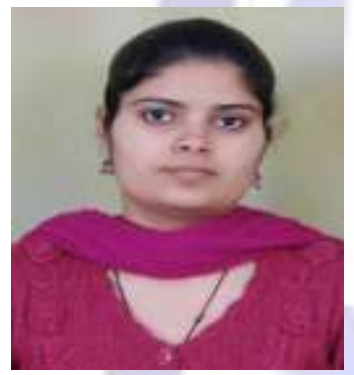

Supreet Kaur received her B.Tech degree in Computer Science from Yadavindra College of Engineering, Talwandi Sabo, in 2010 and pursuing M.Tech degree in Compter Science from Yadavindra College of Engineering, Talwandi Sabo. She was a lecturer with Department of Computer Science, Guru Kashi University; in 2011.Her research interests include digital image processing and real time systems. At present, She is doing her thesis of Mtech in image processing applications.

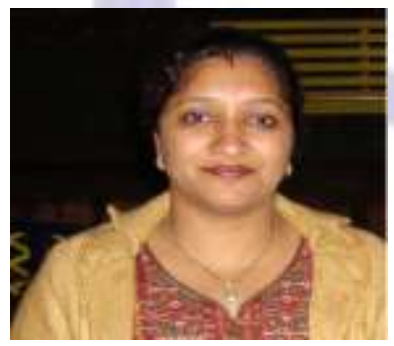

Seema Baghla received her B.Tech degree in Computer Science from IET Bhaddal in 2004 and M.Tech degree in Compter Science from Yadavindra College of Engineering, Talwandi Sabo in 2007. She is an Assistant Professor with Department of Computer Science, YCOE, Guru Kashi Campus, Talwandi Sabo from last five years. Her research interests include digital image processing and Job Sequencing. 